\title{
TUMOR FIBROSO SOLITARIO DEL LÓBULO CAUDADO DEL HÍGADO*
}

\author{
Drs. Marcelo A. Beltrán S. ${ }^{1,2}$, Fernanda Habërle O. ${ }^{1,2}$, Catalina Valenzuela V. ${ }^{1}$, \\ Oriana Urbina C. ${ }^{1}$, Javier Arcos P. ${ }^{2}$, Yamile Haito Ch. ${ }^{1}$ \\ 1 Servicio de Cirugía, Hospital San Juan de Dios de La Serena. \\ 2 Departamento de Clínicas Facultad de Medicina Universidad Católica del Norte. \\ La Serena, Chile.
}

\begin{abstract}
Solitary fibrous tumor of the liver caudate lobe

Introduction: Solitary fibrous tumor of the liver (SFTL) constitutes such a rare finding that it has been published only as case reports and to the present date there are only 45 cases published in English medical literature. This article describes the case of a patient treated at our institution with SFTL. Case report: A male 59-years-old patient was diagnosed with a SFTL incidentally found in a computed abdominal tomography taken by another causes, tumor was originating from the caudate lobe. The patient was asymptomatic. At surgery, the tumor was observed originating directly from the caudate lobe through a pedicle measuring approximately $3 \mathrm{~cm}$. The tumor was resected sectioning its implantation pedicle and ligating all arterial, venous and biliary vessels. The SFTL was well encapsulated, measured $15 \times 9 \times 6 \mathrm{~cm}$ and weighted $794 \mathrm{~g}$. The histological diagnosis was a SFTL and was confirmed by immunohistochemistry with monoclonal antibodies that reacted positively to CD34 and vimentin and negatively to CD117, S100, smooth-muscle $\alpha$-actin and desmin. Conclusions: SFTL is an uncommon tumor. The present case is the first reported in Chilean medical literature and presented all the habitually described radiologic, surgical and pathological characteristics.

Key words: Solitary fibrous tumor of the liver, benign hepatic tumors, liver surgery.
\end{abstract}

\section{Resumen}

Introducción: El tumor fibroso solitario del hígado (TFSH) constituye un hallazgo tan raro que se ha publicado únicamente como reportes de caso y hasta el presente se encontraron sólo 45 casos publicados en la literatura inglesa. El presente artículo describe el caso de un paciente que fue tratado en nuestra institución por un TFSH. Reporte de caso: Paciente masculino de 59 años de edad al que, en una tomografía abdominal computarizada que se tomó por otras causas, se encontró en forma incidental un gran tumor hepático localizado en el lóbulo caudado del hígado. El paciente no refería síntomas relacionados con el tumor. En la cirugía se observó que el tumor se originaba directamente del lóbulo caudado al cual lo unía un pedículo

*Recibido el $1^{\circ}$ de diciembre de 2014 y aceptado para publicación el 14 de abril de 2015.

Los autores no refieren conflictos de interés.

Correspondencia: Dr. Marcelo A. Beltrán S. beltran_01@yahoo.com 
de aproximadamente $3 \mathrm{~cm}$ de diámetro transverso. Se seccionó el tumor desde su base de implantación en el hígado ligándose los vasos arteriales y venosos así como los conductos biliares que se encontraron. Este medía 15 × 9 × $6 \mathrm{~cm}$ y pesaba $794 \mathrm{~g}$, se encontraba bien encapsulado. El diagnóstico histológico fue el de un TFSH, el cual se confirmó mediante inmunohistoquímica con anticuerpos monoclonales que resultaron positivos para CD34 y vimentina en las células tumorales y negativos para CD117, S100, $\alpha$-actina de músculo liso y desmina. Conclusiones: El TFSH es un tumor raro. El presente caso de un TFSH es el primero reportado en la literatura médica chilena y presentó las características radiológicas, quirúrgicas y patológicas habitualmente descritas en estos casos.

Palabras clave: Tumor fibroso solitario del hígado, tumores hepáticos benignos, cirugía hepática.

\section{Introducción}

El tumor fibroso solitario es una neoplasia rara que fue descrita por primera vez el año 1931 por Paul Klemperer y Coleman B. Rabin en un reporte sobre neoplasias de la pleura ${ }^{1}$. Este tumor es habitualmente benigno y ha sido recientemente incorporado por la World Health Organization en su clasificación de tumores de tejidos blandos². El tumor fibroso solitario se encuentra de preferencia en la cavidad torácica, habitualmente en la pleura y mediastino, pero también se ha descrito en otras cavidades serosas como peritoneo y pericardio y en otras localizaciones como el tracto respiratorio superior, las meninges, la cavidad oral, la órbita, la glándula tiroides y tejidos blandos ${ }^{3}$. La localización de esta neoplasia en el hígado es particularmente infrecuente ${ }^{4,5}$. El tumor fibroso solitario del hígado (TFSH) constituye un hallazgo tan raro que se ha publicado únicamente como reportes de caso y hasta el presente año se encontraron sólo 45 casos publicados en la literatura inglesa ${ }^{4-15}$. Las manifestaciones clínicas son inespecíficas, habitualmente referidas al efecto de masa y compresión que el TFSH produce sobre los órganos vecinos ${ }^{4-11}$. El diagnóstico es habitualmente incidental y en algunos casos dirigido durante el estudio radiológico de los síntomas del paciente ${ }^{11-14}$. El tratamiento consiste en la resección tumoral pero el pronóstico no se ha establecido claramente aún debido al limitado número de $\operatorname{casos}^{4-15}$. El diagnóstico definitivo se establece con la inmunohistoquímica debido a que histológicamente presenta características similares a los tumores estromales y mesenquimatosos. La localización más frecuente en el hígado es el parénquima del lóbulo derecho en $60 \%$ de los $\operatorname{casos}^{4} \mathrm{y}$ aun cuando el parénquima del lóbulo izquierdo se encuentra comprometido en $40 \%$ de los pacientes no se han reportado TFSH pediculados que se originen exclusivamente en el lóbulo caudado. El presente artículo describe el caso de un paciente que fue tratado en nuestra institución por un TFSH que se originaba en el lóbulo caudado del hígado.

\section{Reporte de caso}

Paciente de sexo masculino de 59 años de edad con el antecedente patológico de un cáncer de próstata operado el año 2013. Durante el estudio de etapificación, en la tomografía abdominal computarizada (TC), se encontró en forma incidental un gran tumor hepático localizado en el lóbulo caudado del hígado. El paciente no refería síntomas relacionados con el tumor. En la TC se informaba de un tumor polilobulado heterogéneo que medía $10,2 \times 6,9 \times$ $10 \mathrm{~cm}$ y que se originaba del lóbulo caudado del hígado descendiendo por detrás del estómago y por delante del páncreas (Figura 1). El tumor era ligeramente heterogéneo con áreas hiperdensas y algunos sectores hipodensos que no se modificaban con el contraste. La lesión se irrigaba desde una rama de la arteria hepática izquierda (Figura 2). No se visualizaban otras lesiones hepáticas. Los exámenes de laboratorio fueron normales. Con estos estudios se decide la cirugía. El abordaje fue a través de una laparotomía sub-costal bilateral con extensión hacia la línea media superior. La apertura del epiplón menor permitió observar el tumor directamente (Figura $3 \mathrm{~A})$. Este se encontraba por detrás del estómago y por sobre el cuerpo del páncreas sin ninguna adherencia a estos órganos. Se observó que se originaba directamente del lóbulo caudado al cual lo unía un pedículo de aproximadamente $3 \mathrm{~cm}$ de diámetro transverso. Se seccionó el tumor desde su base de implantación en el hígado ligándose los vasos arteriales y venosos así como los conductos biliares que se encontraron. Finalmente se extrajo el tumor sin mayores incidentes. Este medía $15 \times 9 \times 6 \mathrm{~cm}$ y pesaba $794 \mathrm{~g}$, se encontraba bien encapsulado, la cápsula era blanquecina nacarada y de consistencia lisa, el contorno era lobulado y tenía una consistencia firme y esponjosa (Figuras 3B y 3C). Al corte, el tumor reveló una superficie heterogénea, nodular, blanquecina y de consistencia firme. La tinción histoquímica con hematoxilina-eosina informó un tumor de células fusiformes dispuestas en haces cortos entrecruzados con abundantes bandas gruesas de colágeno hialino y áreas focales laxas con 

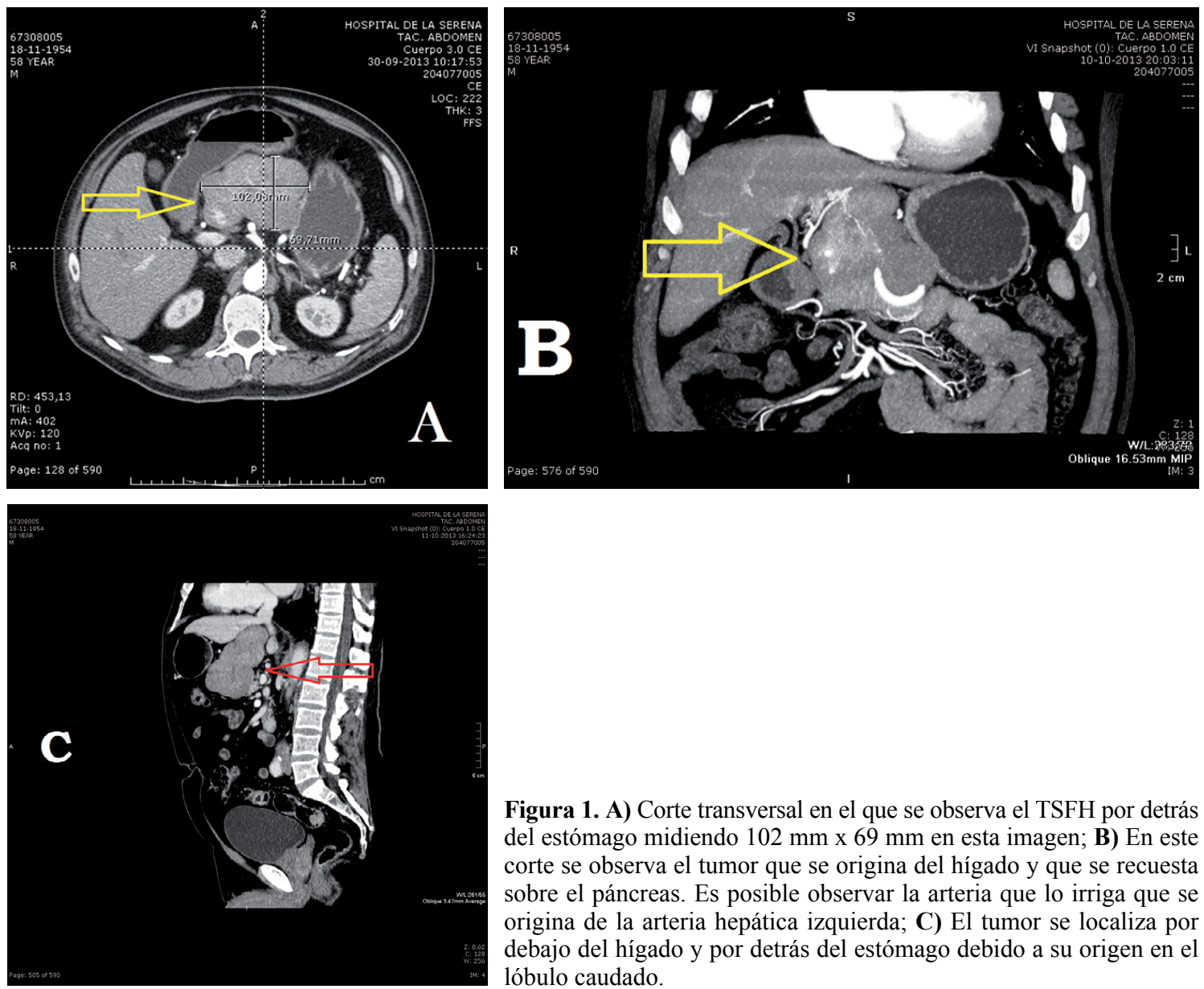

Figura 1. A) Corte transversal en el que se observa el TSFH por detrás del estómago midiendo $102 \mathrm{~mm}$ x $69 \mathrm{~mm}$ en esta imagen; B) En este corte se observa el tumor que se origina del hígado y que se recuesta sobre el páncreas. Es posible observar la arteria que lo irriga que se origina de la arteria hepática izquierda; C) El tumor se localiza por debajo del hígado y por detrás del estómago debido a su origen en el lóbulo caudado.
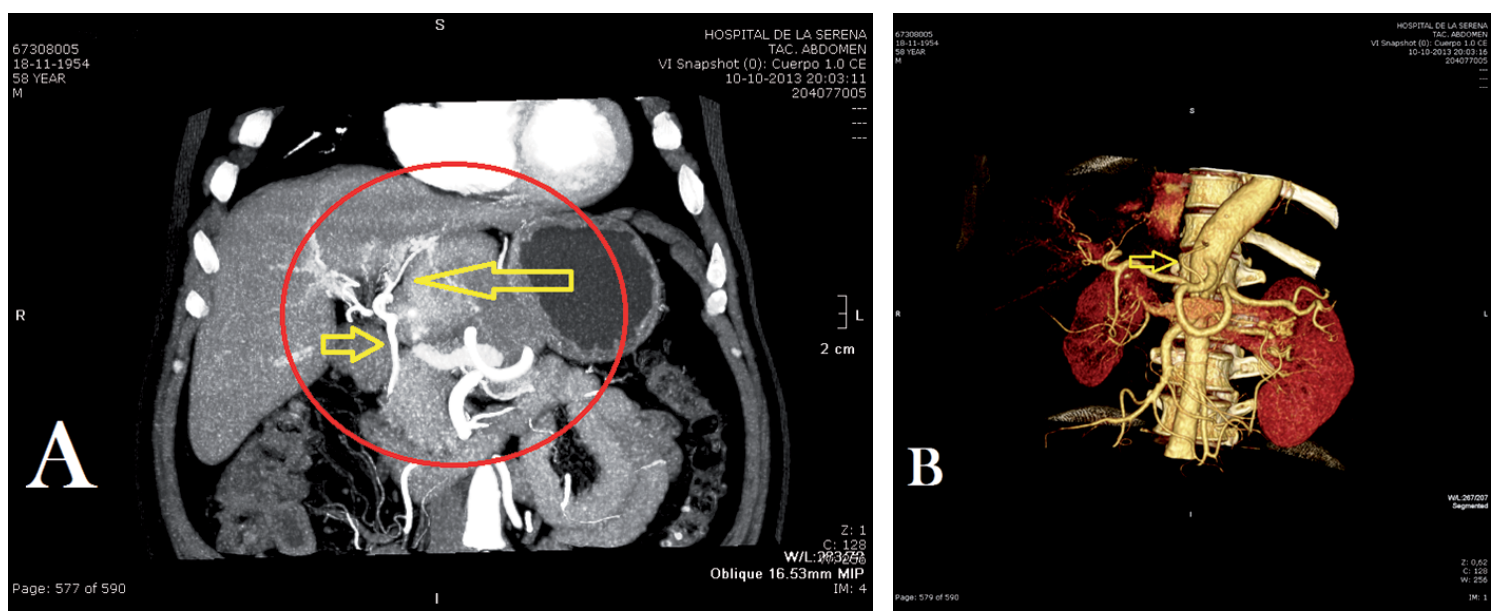

Figura 2. A) Corte oblicuo en fase arterial que permite evaluar la irrigación del tumor y su relación con los vasos del tronco celíaco y la arteria hepática. La flecha pequeña señala la arteria hepática común y la flecha grande la arteria que irriga el TSFH; B) Reconstrucción tridimensional que permite identificar el vaso arterial que irriga el TSFH. 

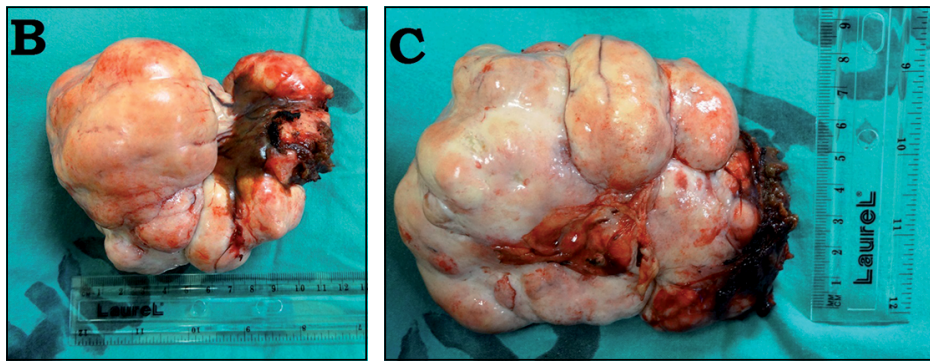

Figura 3. A) Vista del tumor in situ antes de su resección; B) Vista inferior del TSFH, es notable su contorno lobulado y su cápsula de apariencia lisa y nacarada. Es posible observar algo de tejido hepático en el área de su implantación en el hígado; C) Vista superior del TSFH.

escaso infiltrado linfoplasmocitario. No se observó necrosis o atipias. El diagnóstico histológico fue el de un TFSH, el cual se confirmó mediante tinción inmunohistoquímica con anticuerpos monoclonales que resultaron positivos para CD34 y vimentina en las células tumorales y negativos para CD117, S100, $\alpha$-actina de músculo liso y desmina.

\section{Discusión}

Los TFSH son tumores primitivos con diferenciación multidireccional que se originan en el mesénquima y que ocasionalmente comprometen el hígado ${ }^{6,7}$. La mayoría de los TFSH son benignos pero algunos pueden tener un comportamiento maligno $^{6,15}$. Los TFSH se presentan con mayor frecuencia en las mujeres $(72 \%)^{8}$. La edad promedio de presentación es de 55 a 59 años pero se han reportado entre los 16 y 83 años de edad ${ }^{4-16}$. Nuestro paciente, masculino, se encontraba dentro del promedio de edad habitual de presentación de estos tumores. El primer reporte de un TFSH, fue publicado el año 1959 por Donald B. Nevius y Nathan B. Friedman sobre un hombre de 65 años de edad que se presentó con ascitis e hipoglicemia asociadas a un TFSH de comportamiento maligno ${ }^{16}$. Desde entonces, sólo 45 casos más se han reportado, la mayor parte de los cuales fueron TFSH de comportamiento benigno. Notablemente, en este caso, el tumor no se presentó por los síntomas que pudiera ocasionar sino que fue un hallazgo incidental en un estudio radiológico por otra causa. Las manifestaciones clínicas del TFSH son inespecíficas y en general se refieren al efecto de masa que produce el tumor desplazando y comprimiendo los órganos adyacentes ${ }^{5-11}$. La compresión y desplazamiento de órganos y estructuras vecinas se debe al gran tamaño que alcanzan los TFSH que miden entre 5 y $30 \mathrm{~cm}$ de diámetro en el momento de la presentación clínica ${ }^{4-17}$. En algunos casos, incluyendo el caso del primer reporte ${ }^{16}$, el TSFH se presentó asociado a hipoglicemia relacionada a la producción paraneoplásica de Insulin-like Growth Factor tipo II (IGF-II) ${ }^{4}$. Al examen físico es posible palpar una masa firme en la región del hipocondrio derecho o en el epigastrio ${ }^{4-15}$. En este caso, el tumor no fue palpable debido a su localización por debajo del hígado y detrás del estómago. El hallazgo de una masa tumoral lleva habitualmente al estudio radiológico, inicialmente con una ecografía, continuando con una TC o resonancia magnética (RM). Los hallazgos ecográficos muestran tumores heterogéneos levemente hiperecoicos en relación al parénquima hepático normal, con o sin calcificaciones ${ }^{11,17}$. La heterogeneidad demostrada representa áreas de degeneración mixoide ${ }^{12}$. Aun cuando los TSFH son tumores muy vascularizados, no es posible demostrar flujo con el Doppler ${ }^{12}$. En la TC estos tumores aparecen como masas heterogéneas con áreas de aumento de densidad irregular durante la fase portal ${ }^{11,17}$. Las imágenes en la RM en T1 muestran tumores heterogéneos hipodensos en relación al tejido hepático normal ${ }^{11,13,17}$. Las imágenes T2 muestran áreas hipodensas e hiperdensas en un patrón que se ha descrito como mixto blanco-negro debido al contenido de colágeno ${ }^{12,13,17}$. La cápsula tumoral se define más claramente en las imágenes de la RM que en las imágenes de la $\mathrm{TC}^{12}$. Todas estas características radiológicas se observaron en la TC de este paciente. La resección hepática de tumores benignos es segura y se recomienda en pacientes seleccionados con tumores benignos no-quísticos ${ }^{18,19}$. El tratamiento recomendado para el TFSH es la resección quirúrgica debido al potencial de malignidad que tiene ${ }^{14,20}$. Este caso fue operado mediante laparotomía y resección de la base de implantación en el lóbulo caudado hepático; no requirió de una hepatectomía formal. Macroscópicamente, el TFSH es un tumor bien encapsulado con una superficie 
lisa de color gris-blanquecino ${ }^{7,14}$. El diagnóstico definitivo se basa en la asociación de características histológicas e inmunohistoquímicas. La estructura histológica de estos tumores consiste en la presencia de abundantes células fusiformes arregladas en un patrón estoriforme intercaladas con áreas acelulares compuestas por gruesas bandas hialinas de colágeno y vasos sanguíneos similares a los que se presen$\tan$ en los hemagiopericitomas ${ }^{8-10,14,17}$. Los TFSH reaccionan positivamente a la tinción con CD34, CD99, vimentina y Bcl-24,8,9,14,17. Son negativos para CD117 los que los diferencia de los tumores estromales, también reaccionan negativamente a la $\alpha$-actina de músculo liso, desmina, citokeratina y S-100 lo que los diferencia de los leiomiomas, fibrosarcomas, hemagioendoteliomas y tumores de origen en la vaina neural ${ }^{4,5,9,14}$. El pronóstico histológico está dado por la actividad mitótica, pleomorfismo celular, Ki-67 menor a 5\%, presencia de necrosis e invasión local ${ }^{6,8,17}$. El TFSH de este paciente fue positivo a los marcadores inmunohistoquímicos habituales los que confirmaron el diagnóstico. El tumor tiene habitualmente un comportamiento benigno, sin embargo, existe un potencial de malignización aún no completamente estudiado ni caracterizado. En los 45 casos conocidos se encontraron 2 tumores malignos (4\%), los que constituyen los únicos casos documentados malignos ${ }^{15,16}$.

El presente reporte, discute un TFSH del lóbulo caudado, que constituye además una presentación atípica de este tumor hepático. Todas las otras características habitualmente descritas en estos tumores se presentaron en este caso.

\section{Referencias}

1. Klemperer P, Rabin CB. Primary neoplasms of the pleura: A report of five cases. Arch Pathol. 1931;11:385-412.

2. Fletcher CD. The evolving classification of soft tissue tumors: An update based on the new WHO classification. Histopathology 2006;48:3-12.

3. Vallat-Decouvelaere AV, Dry SM, Fletcher CD. Atypical and malignant solitary fibrous tumors in extrathoracic locations: Evidence of their comparability to intrathoracic tumors. Am J Surg Pathol. 1998;22:1501-11.

4. Ji Y, Fan J, Xu Y, Zhou J, Zeng HY, Tan YS. Solitary fibrous tumor of the liver. Hepatobiliary Pancreat Dis Int. 2006;5:151-3.

5. Vennarecci G, Ettorre GM, Giovannelli L, del Nonno F, Perracchio L, Visca P, et al. Solitary fibrous tumor of the liver. J Hepatobiliary Pancreat Surg. 2005;12:341-4.

6. Perini MV, Herman P, D'Albuquerque LA, Saad WA. Solitary fibrous tumor of the liver: Report of a rare case and review of the literature. Int J Surg. 2008;6:396-9.

7. Famá F, Le Boue Y, Barrande G, Villeneuve A, Berry MG, Pidoto RR, et al. Solitary fibrous tumor of the liver with IGF-II-related hypoglycemia: A case report. Langenbecks Arch Surg. 2008;393:611-6.

8. Sun K, Lu JJ, Teng XD, Ying LX, Wei JF. Solitary fibrous tumor of the liver: A case report. World J Surg Oncol. 2011;9:37.

9. Korkolis DP, Apostolaki K, Aggeli C, Plataniotis G, Gontikakis E, Volanaki D, et al. Solitary fibrous tumor of the liver expressing CD34 and vimentin: A case report. World J Gastroenterol. 2008;14:6261-4.

10. Liu Q, Liu J, Chen W, Mao S, Guo Y. Primary solitary fibrous tumors of liver: A case report and literature review. Diag Pathol. 2013;8:195.

11. Fuksbrumer MS, Klimstra D, Panicek DM. Solitary fibrous tumor of the liver: Imaging findings. Am J Radiol. 2000;175:1683-7.

12. Ginat DT, Bokhari A, Bhatt S, Dogra V. Imaging features of solitary fibrous tumors. Am J Radiol. 2011;196:487-95.

13. Soussan M, Felden A, Cyrta J, Morere JF, Douard R, Wind P. Solitary fibrous tumor of the liver. Radiology 2013;269:304-8.

14. Novais P, Medranda CR, Pannain VL, Barbosa D, Biccas B, Fogaca H. Solitary fibrous liver tumors: Is surgical approach the best option? J Gastrointest Liver Dis. 201;19:81-4.

15. Jakob M, Schneider M, Hoeller I, Laffer U, Kaderli R. Malignant solitary fibrous tumor involving the liver. World J Gastroenterol. 2013;19:3354-7.

16. Nevius DB, Friedman NB. Mesotheliomas and extraovarian thecomas with hypoglycemic and nephrotic syndromes. Cancer 1959;12:1263-9.

17. Taboada V, Zueco C, Sobrido C, Martínez V. Tumor fibroso solitario hepático: Hallazgos radiológicos y revisión de la bibliografía. Radiología 2010;52:67-70.

18. Ibrahim $\mathrm{S}$, Chen $\mathrm{CL}$, Wang $\mathrm{SH}$, Lin $\mathrm{CC}$, Yang $\mathrm{CH}$, Yong CC, et al. Liver resection for benign liver tumors: Indications and outcome. Am J Surg. 2007;193:5-9.

19. Clarke DL, Currie EJ, Madhavan KK, Parks RW, Garden OJ. Hepatic resection for benign non-cystic liver lesions. HPB 2004;6:115-9.

20. Moran CA, Ishak KG, Goodman ZD. Solitary fibrous tumor of the liver: a clinicopathologic and immunohistochemical study of nine cases. Ann Diagn Pathol. 1998;2:19-24. 